\title{
Design of remote monitor software based on C\# and XML
}

\author{
Gao-Feng Pan, Sheng Liang, Si-Fang Liu ${ }^{\dagger}$ and Yong Xie \\ China Satellite Maritime Tracking and Control Department, \\ Jiangyin 214431, China \\ liumaster2006@163.com \\ ${ }^{\dagger}$ Corresponding author: Si-Fang Liu
}

\begin{abstract}
The monitoring terminal is an important part, which is interactive with users in TT\&C equipment remote monitoring system, but it has some problems such as more work patterns, data process, and data display, etc. The work patterns and state parameter is designed by XML file, and data in XML file is used to be read and written with the advantages of XML, meanwhile achieve the function flexible by different XML file. Obtain good effect by this method, not only reduce the software workload, but also improve the code efficiency.
\end{abstract}

Key words: Monitor terminal; XML; Class relation; Multithreading

\section{Introduction}

For real-time inter-bank ship cannot carry out technical exchanges, coordination of technical problems long cycle, low efficiency equipment troubleshooting, design a shipboard control equipment remote monitoring system, not only to facilitate land timely, accurate information on shipborne terminal equipment working conditions, but also for tracking ship to provide efficient, fast technical support and security, and remote monitoring terminal is an important direct user-oriented.

Using XML files for network data analysis, storage and control method of dynamic assignment, monitoring terminal design is implemented in c\# environment, flexible solution to multi-parameter data, display problems, get better practical effect.

\section{Remote Monitoring System Principles}

Monitoring and control equipment remote monitoring system is divided into "ship terminal" and "land-side" in two parts, system block diagram shown in Figure 1. Shipborne end remote diagnostics test server, complete with interactive system monitoring device status information; information exchange server, complete communication protocol conversion services, data transmission 
communication, file transfer, etc. Relay. Land end remote monitoring and control equipment testing server to receive and send all kinds of information on land side, the device information stored in the database and the information is classified; remote monitoring terminal to complete analytic data display, to monitor the status of shipborne equipment to provide a visual interface, is an important component of direct user-oriented, but also partially set forth herein.

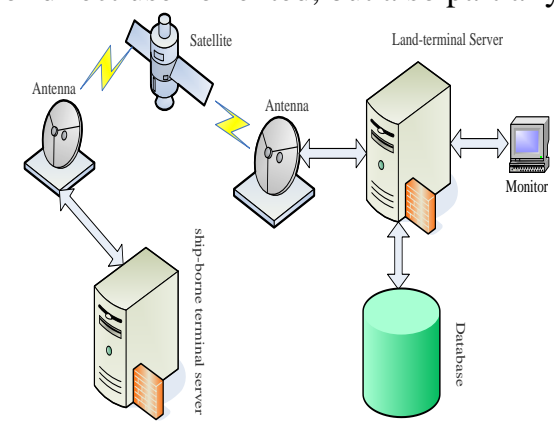

Fig.1The diagram of system block

\section{Design of Monitor Terminal}

In figure 2, shows that more monitored objects required to monitor Terminal, monitor including configuration information parameters, equipment operating status information such as tens of thousands. Moreover, even in the same device, there are different modes correspond to different working conditions; working mode or State of the same device, some parameters have the same meaning. In software design, the logic is more complex, if we adopt the structure for data processing, a large amount of coding and is not conducive to the upgrade and maintenance of the system. Therefore, full use of the advantages of XML technology, design, the work of device models, parameter configuration designed XML file in the form[1], and as part of the background processing XML files, not only reducing the workload and improve the efficiency of the code. Realization of a network as the channel, based on XML technology as the core, monitoring terminal based on XML configuration file parameters. 


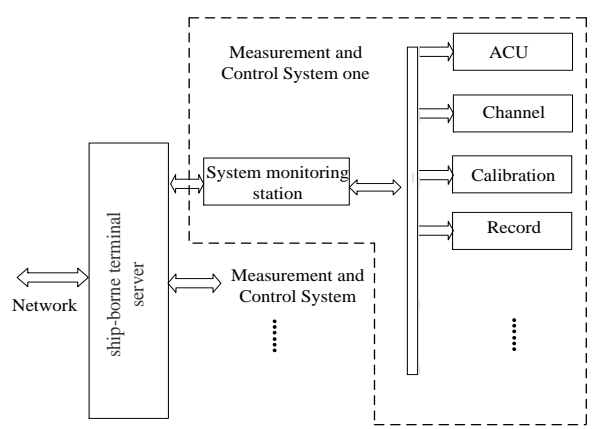

Fig2. The diagram of monitoring terminal

\section{Software Features}

Monitoring software used to parse, display the data received and the subsequent data playback, including real time data processing module, display module, user interface, data playback module [2], the software process as shown in Figure 3.

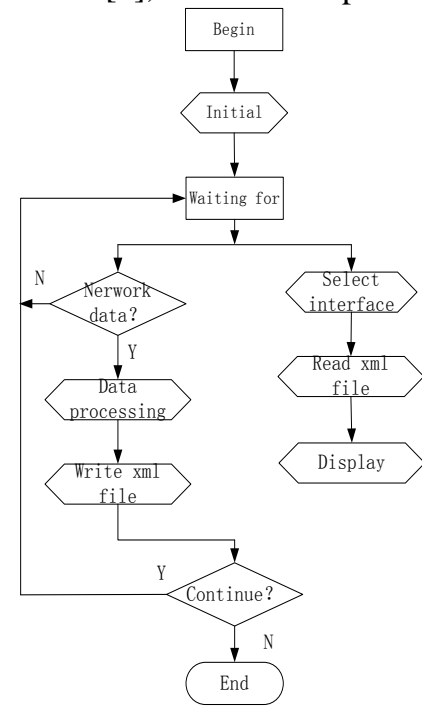

Fig3. The flow chart of software

Each module function is introduced as follows:

(1) data processing module Mainly complete the receiving data from the server side, in accordance with the agreement format parse the data, and provides the software and data interaction between XML documents, realize data storage and reading;

(2) real-time display module will the data in the XML file, according to the specified way for processing and display; 
(3) the user interface between the user and the software system interface module, data display and receives the user's operation;

(4) data playback module Read the test server database file information, and shall be carried out in accordance with the time sequence data playback or chart shows.

\section{Software Design}

\section{(1)Class relationships}

Software development as the core module, data processing module in the design data and code together, for different modules, management operation, reduce the mutual influence between modules, the software structure is clear, simple maintenance; Software startup configuration XML data into memory, the data interaction between the various modules in memory processing, improve the utilization rate of resources and data processing speed, as shown in figure 4, the basic relationship between its core as XML, speaking, reading and writing class.

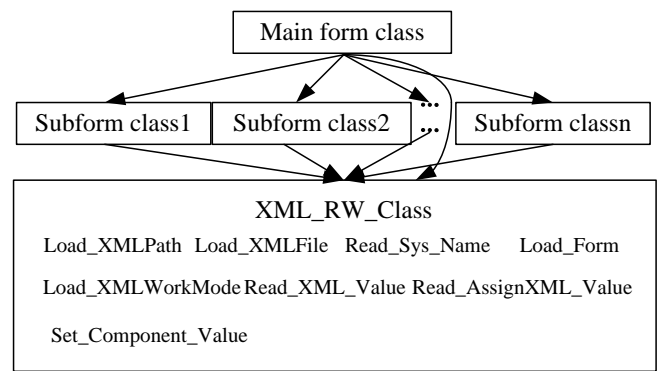

Fig4.The class diagram of monitoring terminal software

In figure 4, Set_XMLValue parse () method for receiving data and deposit to the XML file; Read_XMLName () method to find the specified XML file and variable number, device name, etc. Read_XMLValue () method of the complete XML file to read and real-time display.

\section{(2) The XML file to read and write}

In XML files as data configuration file, the realization of software interaction of external and internal data, make the core of the XML configuration files as data sources, all data storage and interaction are done by XML files and data types include two types: the I/O and A/D amount, the corresponding XML file describes the sample is shown in figure5: 


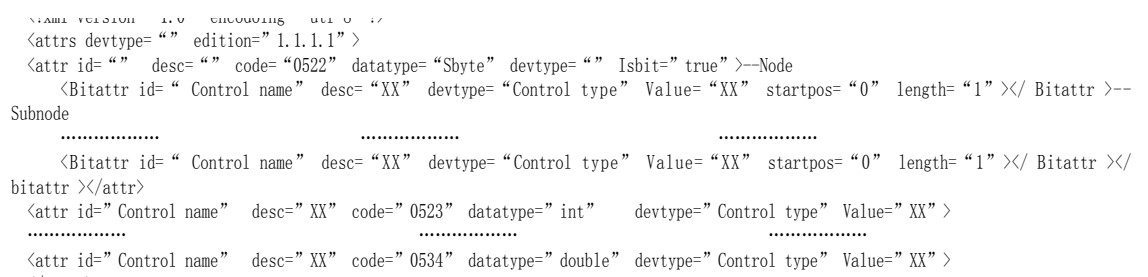

Fig5. The diagram of XML file describing

We can know from the sample XML file tree structure and clear description, very easy to implement the data frame processing. XML has high expansibility, just modify the corresponding bytes or child, and don't need to modify the code content to adapt to changes in data frames; XML can support different compiler environment, easy to realize cross-platform operation, etc., based on the above points in this paper, using XML technology to manipulate the data frame.

Monitoring terminal receives the network or database data, according to the format of the data structure, first of all, according to the variable code to distinguish the different equipment data, then according to the variable coding and data field contains the length of the data to obtain the data, and stored in the specified XML file, at the same time, using the C\# language[3], multithreading and timer processing real-time read different equipment corresponding XML files, equipment data obtained and displayed to the interface.

\section{(3) The window fusion technology}

The interface of the system needed to show nearly 50, interface display processing technology is particularly important. In C\# using the panel controls to complete different form called, using the specified parent container method [4], the example code is as follows:

Form2 form=new Form2();

form.MdiParent=this;

form.Parent=this.Panel1;

Frm.FormBorderStyle $=$ FormBorderStyle.None;

panelform.Show();

(4) Interface controls dynamic assignment

Because the monitoring parameter is more, interface controls respectively assignment will increase the amount of compiled code, in this paper, we use XML file defines the control method and control types, interface control was achieved by using control traversal methods assignment [5], the process is shown in figure 6: 


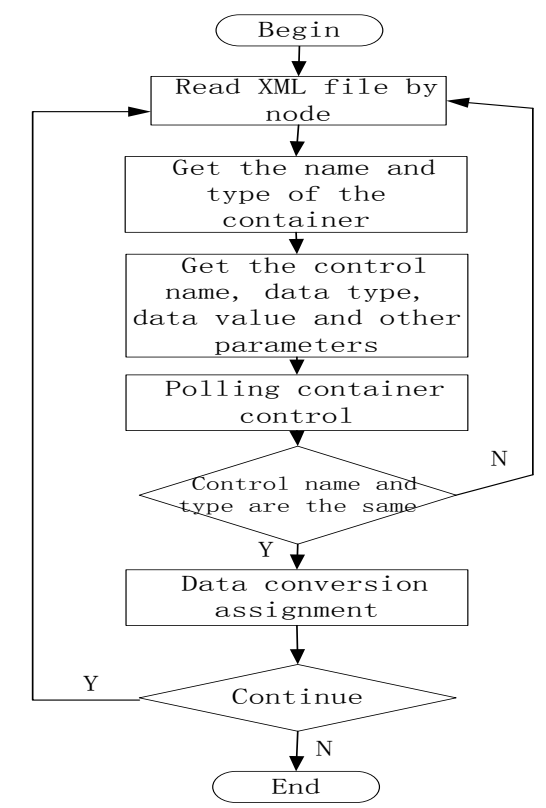

Fig6. The flow chart of interface controls dynamic assignment

(5) Across the form values

In the main forms invoke other form, because it is associated with the XML file, it will need to stay a particular value to open the window. In this paper, through the form of the public attribute values across form the values, the example code is as follows:

In the form Form 2 defines a public property

$\cdots$

The get

\{

Return parameters;

\}

The set

\{

Parameter $=$ value;

\}

In the form Form1 such calls

Form2 f2 = new Form2 ();

F2. Parameter $=\mathrm{XXX}$; 


\section{Summary}

Using XML file to realize the real-time monitoring the working state of measurement and control instruments from a ship, the software runs stability, control performance is good. By actual test, the proposed XML file as a monitoring software method to realize the data source, data processing speed is high, stable running, simple, practical, and has achieved expected effect of satisfaction. A follow-up study, further optimize data storage structure and algorithms, to improve software performance.

\section{References}

[1] Hao Zhaoping. Study on XML Application and Development Trend [J]. Application of the technology for Computer Engineering, 2009(9). (In Chinese)

[2] Wang Zhenjiang. XML Programming. Beijing: China Railway Publishing House, 2006. (In Chinese)

[3] Christian Nagel, Bill Evjen, Jay Glynn[USA], Translated by Li Ming. Advanced Programming C\# (7th edition).Beijing: Tsinghua University Press, 2010. (In Chinese)

[4] Li Qiuyun.XML and WML Tutorial. Beijing: People's Posts and Telecommunications Press, 2001. (In Chinese)

[5] Feng Tinghui. XML Completely Manual [M] Beijing: Chinese Power Press, 2000. (In Chinese) 